\title{
Óleos essenciais de espécies vegetais reduzem a germinação de capim
} annoni

\author{
Essential oils of plant species reduce the lovegrass germination
}

\author{
Joseila Maldaner \\ Gerusa Pauli Kist Steffen
}

Centro de Pesquisa em Florestas - Departamento de Diagnóstico e Pesquisa Agropecuária do Rio Grande do Sul Santa Maria - Rio Grande do Sul - Brasil

\section{Ricardo Bemfica Steffen}

Empresa Biotec RS Tecnologia e Consultoria-Santa Maria - Rio Grande do Sul - Brasil

Evandro Luiz Missio

Cleber Witt Saldanha

Rosana Matos de Morais

Centro de Pesquisa em Florestas - Departamento de Diagnóstico e Pesquisa Agropecuária do Rio Grande do Sul -

Santa Maria - Rio Grande do Sul - Brasil

Tamires Silveira Moro

\section{Resumo}

Universidade Federal de Santa Maria - UFSM - Santa Maria - Rio Grande do Sul - Brasil
O capim annoni (Eragrostis plana Nees) é uma gramínea tropical, perene, nativa do sudoeste da África, que foi introduzida acidentalmente, em meados dos anos 1950, e, a partir de sua propagação, tornou-se uma importante planta invasora nos estados do sul do Brasil devido suas características de alta competitividade e difícil controle, comprometendo a qualidade das pastagens naturais pertencentes ao Bioma Pampa. Assim, o objetivo deste estudo foi analisar o potencial de algumas espécies aromáticas sobre a germinação do capim annoni. Diferentes concentrações do óleo essencial de alecrim, canela, carqueja, capim limão, cipreste, citronela, eucalipto citriodora, eucalipto grandis, melaleuca, louro e pinus foram testados como solução umedecedora do papel de germinação em caixas do tipo gerbox. Foram coletados dados de primeira contagem, germinação acumulada e calculado o índice de velocidade de germinação. Os óleos das diferentes espécies variaram quanto ao potencial de inibição da germinação, essa variação também foi observada em respostas às concentrações testadas. Destacaram-se no controle da germinação de capim annoni os óleos de melaleuca, carqueja e louro, indicando que são promissores para a formulação de produtos para a contenção da dispersão desta espécie invasora.

\section{Abstract}

Lovegrass (Eragrostis plana Nees) is a perennial tropical grass native to southwestern Africa, which was introduced accidentally in the 1950s and, since its spread, has become a major invasive plant in the south of Brazil due to its characteristics of high competitiveness and difficult control, compromising the quality of the natural pastures belonging to the Pampa Biome. Thus, the objective of this study was to analyze the potential of some aromatic species on lovegrass germination. Different concentrations of essential oil of rosemary, cinnamon, carqueja, lemongrass, cypress, citronella, eucalyptus citriodora, eucalyptus grandis, melaleuca, bay laurel and pinus were tested as a wetting solution of germination paper in boxes of gerbox type. Data of first count, accumulated germination and germination rate index were calculated. The oils of the different species varied in the potential of germination inhibition, this variation was also observed in responses to the concentrations. Melaleuca, carqueja and laurel oils were highlighted in the control of the lovegrass germination, indicating that they are promising for the formulation of products to contain the dispersion of this invasive species.

\section{Palavras-chave}

Eragrostis plana. Metabólitos secundários. Planta invasora. Bio-herbicida. Controle alternativo.

\section{Keywords}

Eragrostis plana. Secondary metabolites. Invasive plant.

Bioherbicide. Alternative control. 


\section{Introdução}

Eragrostis plana Nees, popularmente conhecida como capim annoni, é uma espécie da família Poaceae, tropical, perene, estival, originária da África (Ferreira et al., 2008). Foi introduzida no Rio Grande do Sul na década de 1950, como contaminante de sementes de Chloris gayana Kunth, também conhecido por capim Rhodes (Zenni \& Ziller, 2011). Inicialmente, acreditou-se ser uma potencial planta forrageira devido a sua tolerância às flutuações do clima, especialmente à geada, e assim foi reproduzida e comercializada na forma de semente pelo Grupo Rural Annoni para várias regiões do estado e do país (Goulart et al., 2009; Ferreira, 2011a). No entanto, após avaliação agronômica, essa espécie mostrou-se inapta para utilização em pastejo devido à baixa qualidade nutricional e à elevada resistência à tração mecânica, resultando em baixa produção animal (Alfaya et al., 2002; Medeiros \& Focht, 2007). Mesmo em seu local de origem, o capim annoni é considerado uma planta indesejável em função das características que o tornam pouco palatável aos animais e favorecem sua dispersão e perpetuação em biomas campestres ou savanas (Kirkman \& Morris, 2003).

A alta rusticidade, adaptação a solos pobres e grande capacidade de multiplicação por sementes, conferem ao capim annoni destaque entre as espécies exóticas invasoras de maior impacto (Goulart et al., 2009). Estes atributos tornam a espécie de fácil dispersão e de difícil controle, chegando a infestar cerca de $20 \%$ da vegetação dos campos do bioma Pampa na região Sul do Brasil, o que equivale a 3,1 milhões de hectares (Goulart et al., 2009; Cicconet et al., 2015). Este alto grau de infestação também é responsável por prejuízos econômicos de grande relevância, sendo que num período de 10 anos (1996-2005), essas perdas foram estimadas em US\$ $88.500 .000,00$, somente considerando a falta de produção das áreas invadidas. No entanto, se forem consideradas as arrecadações governamentais que seriam geradas pela produção animal nas áreas improdutivas infestadas pelo capim anonni, as perdas seriam ainda mais surpreendentes (Focht, 2008).

A contenção da dispersão desta espécie é praticamente restrita ao controle químico pelo uso de herbicidas (Nachtigal et al., 2009), o que ambientalmente não é uma estratégia interessante, pois pode causar variações na diversidade das populações de espécies invasoras e acentuar contaminação do meio ambiente.

Os metabólitos secundários liberados pelas plantas no ambiente podem influenciar positiva ou negativamente o crescimento e desenvolvimento da vegetação adjacente. A essa relação dá-se o nome alelopatia (Farooq et al., 2011). Neste sentido, a compreensão das interações alelopáticas relacionadas aos compostos de origem natural, oriundos do metabolismo secundário de plantas, podem oferecer novas e importantes alternativas para o manejo de plantas espontâneas e por isso vêm sendo alvos de pesquisas.

Diversos constituintes químicos isolados de espécies vegetais, em especial os óleos essenciais, têm demonstrado efeito sobre fungos fitopatogênicos como Colletotrichum gloeosporioides Penz., Fusarium oxysporum Schltdl. e Alternaria solani Ell. \& Mart. (Takatsuka et al., 2003; Balbi-Peña et al., 2006), e atividade bioherbicida para várias espécies de plantas daninhas, como Achyranthes aspera Wall., Cassia occidentalis L., Parthenium hysterophorus L., Echinochloa crusgalli L. e Ageratum conyzoides L. (Ens et al., 2009). No entanto, não há registros na literatura de estudos que avaliaram o efeito de compostos vegetais para a inibição do capim annoni. Assim, o objetivo deste trabalho foi avaliar o efeito de óleos essenciais de espécies vegetais sobre a germinação do capim annoni.

\section{Material e métodos}

Para os ensaios deste trabalho, foi utilizado um lote composto por sementes de capim annoni coletadas em diferentes cidades da região Centro-Sul do Rio Grande do Sul (Hulha Negra, Dom Pedrito, São Gabriel e Santa Maria) em áreas de abrangência do bioma Pampa. As 
sementes foram armazenadas em geladeira $( \pm 4 \stackrel{\circ}{\circ})$, onde permaneceram até o momento da instalação dos ensaios.

\section{Extração dos óleos essenciais}

Foram estudados os óleos essenciais de alecrim (Rosmarinus officinalis L.), canela (Cinnamomum zeylanicum Blume), carqueja (Baccharis trimera Less.), capim limão (Cymbopogon citratus Stapf.), cipreste (Cupressus sempervirens L.), citronela (Cymbopogon winterianus Jowitt), eucalipto citriodora (Corymbia citriodora Hook), eucalipto grandis (Eucalyptus grandis W. Mill), melaleuca (Melaleuca alternifolia Cheel), pinus (Pinus elliottii Engelm.) e louro (Laurus nobilis L.).

O processo de extração dos óleos essenciais seguiu a técnica de hidrodestilação por arraste de vapor, utilizando-se folhas frescas, segundo metodologia adaptada de Vitti \& Brito (2003). Os óleos essenciais extraídos, após a passagem por um condensador, foram coletados, armazenados em microtubos do tipo eppendorf e mantidos a $4{ }^{\circ} \mathrm{C}$ até sua utilização.

Os ensaios com os diferentes óleos foram independentes, no entanto as diluições para composição dos tratamentos (concentrações do óleo) foram iguais para todas as espécies testadas. Os óleos foram diluídos em álcool etílico 99\% (1:1 - v:v) antes da composição dos tratamentos para possibilitar a homogeneização em solução aquosa. Os experimentos consistiram de sete tratamentos cada: controle (água destilada); controle álcool; 0,001; 0,005; 0,$01 ; 0,05$ e $0,1 \%$ (v/v) do óleo essencial diluído em álcool. O controle álcool foi estabelecido com a maior concentração de álcool utilizada na diluição, a fim de se descartar o efeito do álcool nos tratamentos.

\section{Instalação dos experimentos}

Após um leve processo de desinfestação feito com água destilada, hipoclorito (2,5\%), detergente comercial e álcool 70\%, as sementes foram dispostas sobre papel de germinação do tipo mata-borrão em caixas do tipo gerbox (capacidade $250 \mathrm{~mL}$, medida $11 \times 11 \times 3,5 \mathrm{~cm}$ ). 0 papel de germinação foi umedecido com a solução respectiva de cada tratamento em um volume de $5 \mathrm{~mL}$ por repetição, sendo reaplicados $2 \mathrm{~mL}$ a cada quatro dias para evitar a desidratação. Foram utilizadas quatro repetições por tratamento. Cada repetição consistiu de uma caixa do tipo gerbox com 25 sementes de capim annoni. Os testes foram realizados em sala de crescimento climatizada, com temperatura controlada de 25 ㅇ $\mathrm{C} \pm 2$ e fotoperíodo de 12 horas. Para fins de padronização, considerou-se como semente germinada aquela que emitiu radícula de, no mínimo, $2 \mathrm{~mm}$ de comprimento. Foram avaliadas as seguintes variáveis:

Primeira contagem de germinação - Foi realizada a contagem e retirada das sementes germinadas aos sete dias após a incubação juntamente da primeira avaliação de germinação acumulada, adaptando-se a metodologia descrita por Brasil (2009). Os resultados foram expressos em porcentagem de germinação.

Germinação acumulada - Foi realizada a contagem total de sementes germinadas aos 14 dias após a incubação, adaptando-se a metodologia descrita por Brasil (2009). Os resultados foram expressos em porcentagem de germinação.

Índice de velocidade de germinação (IVG) - Efetuado com anotações diárias do número de sementes germinadas até sete dias após a incubação. Após o término das leituras, o IVG foi calculado pela seguinte fórmula: IVG $=\mathrm{G} 1 / \mathrm{N} 1+\mathrm{G} 2 / \mathrm{N} 2+\ldots \mathrm{Gn} / \mathrm{Nn}$, onde: IVG = índice de velocidade de germinação; $G 1, G 2, \ldots \mathrm{Gn}$ = número de plântulas normais computadas na primeira contagem, na segunda contagem e na última contagem; $N 1, N 2, \ldots \mathrm{Nn}=$ número de dias da semeadura à primeira, segunda e última contagem (Maguire, 1962).

Os dados de porcentagem foram previamente submetidos à transformação arco seno a fim de atender às pressuposições do modelo matemático. Em seguida, todas as variáveis 
foram submetidas à análise da variância, sendo as médias comparadas pelo teste de Tukey ( $P$ $<0,05)$ com a ajuda do programa estatístico SISVAR 5.6 (Ferreira, 2011b).

\section{Resultados e discussões}

Os óleos essenciais da maioria das espécies testadas apresentaram efeito alelopático sobre a germinação das sementes de Eragrostis plana (Tabelas 1, 2 e 3), isso se deve ao conhecido papel de alguns metabólitos secundários vegetais como agentes aleloquímicos na germinação de outras espécies. Esta interferência na germinação que algumas espécies têm sobre as outras pode ocorrer através de diferentes mecanismos, por exemplo, podem interferir no balanço dos fitormônios que agem na divisão e/ou no alongamento celular, na síntese de ácido nucleico e proteínas, na quantidade de oxigênio que chega ao embrião, na permeabilidade das membranas e na inibição da fotossíntese (Taylor \& Grotewold, 2005; Weir et al., 2004).

A primeira contagem de germinação das sementes de capim annoni foi reduzida significativamente, por três dos onze óleos testados. Os óleos de carqueja, louro e melaleuca inibiram a germinação de capim annoni quando adicionados ao papel substrato na concentração de $0,1 \%$, sendo que o óleo de melaleuca também reduziu significativamente a porcentagem de sementes germinadas nas concentrações de 0,01 e 0,05\%. Embora tenha havido redução da germinação com a adição de outros óleos, como por exemplo, canela e citronela, esta não foi diferente da redução provocada pelo controle álcool (Tabela 1). Entre as espécies cujas concentrações dos óleos não influenciaram a primeira contagem de germinação de capim annoni estão capim limão, cipreste, as duas espécies de eucalipto e pinus (Tabela 1).

Quando se considerou a germinação acumulada, foram eficientes em reduzir a germinação de capim annoni os óleos essenciais de alecrim, carqueja, louro e melaleuca, especialmente nas concentrações de 0,05 e $0,1 \%$. Com a aplicação do óleo de melaleuca foi possível atingir uma redução de até $54,56 \%$ na germinação acumulada (Tabela 2). Já os óleos de carqueja, louro e alecrim reduziram a germinação acumulada em 47,25, 38,58 e 20,38\%, respectivamente (Tabela 2 ).

Os resultados observados demonstram que as respostas aos agentes aleloquímicos são espécie-específicas, além de serem também dependentes da concentração do agente aplicado. Weir et al. (2004) também já haviam reportado a importância em se avaliar concentrações nas relações alelopáticas. Agregando a essa ideia, Souza Filho et al., (2009) relatam que a atividade biológica de um determinado agente aleloquímico depende tanto da concentração do mesmo quanto do limite de resposta da espécie tratada, limite este que é relacionado à sensibilidade da espécie receptora, ao metabolismo da planta e às condições ambientais.

Ferreira e Borghetti (2004) apontam que o efeito alelopático pode não acontecer sobre a germinabilidade (percentual final de germinação), mas sobre a velocidade de germinação. Neste sentido, foi verificado que três das onze espécies testadas promoveram redução no índice de velocidade de germinação (IVG) de capim annoni. As maiores reduções na velocidade de germinação foram observadas com o uso dos óleos de carqueja, melaleuca e citronela, principalmente nas maiores concentrações aplicadas (Tabela 3). Por outro lado, os óleos de alecrim, canela, capim limão, cipreste, eucalipto citriodora, eucalipto grandis, louro e pinus não alteraram significativamente a velocidade de germinação de capim annoni, considerandose os dois controles.

Embora a redução da velocidade de germinação represente um atraso na emergência das plântulas, o que é um fator interessante, a inibição da germinação acumulada é uma resposta mais efetiva, quando o objetivo é o controle de espécies invasoras, ou seja, o resultado final de um menor número de indivíduos germinados indica um controle mais definitivo do que apenas um atraso no processo de germinação. Assim, a repercussão prática dos resultados dos testes representados neste trabalho implica que, no ambiente, o 
impedimento da germinação de sementes quimicamente sensíveis a substâncias alelofitotóxicas pode ter como consequência a diminuição da densidade de seus indivíduos, o que poderia levar ao completo controle da dispersão da espécie invasora, caso fosse adotado um manejo que previsse a aplicação periódica e planejada de óleos essenciais das referidas espécies.

Nas concentrações testadas, nenhum dos óleos suprimiu na totalidade a germinação de capim annoni. No entanto, os resultados permitem destacar algumas espécies mais efetivas em inibir a germinação desta invasora, como por exemplo, os óleos de melaleuca, carqueja e louro.

Trabalhos anteriores já indicaram a potencialidade dessas espécies na inibição da germinação de outras. Segundo descrito por Depiné (2003), o extrato da parte aérea de carqueja (Baccharis trimera), apresentou efeito alelopático sobre sementes de picão-preto (Bidens pilosa L.), reduzindo a porcentagem e a velocidade de germinação. Além disso, a dose usual do chá de carqueja aumentou o índice de anomalias do ciclo mitótico e proporcionou alterações cromossômicas em células de Allium cepa, demonstrando efeito alelopático importante no desenvolvimento da espécie testada (De Pinho et al., 2010).

No presente estudo, o óleo de carqueja mostrou-se eficaz na inibição da germinação de capim annoni, provavelmente devido a suas características fitoquímicas. A análise fítoquímica de carqueja constatou a presença de flavonoides, taninos, ácidos graxos, esteroides e/ou triterpenoides, cumarinas, aminogrupos e traços de glicosídeos saponínicos (Bona et al., 2002). Alguns autores atribuem aos taninos os efeitos supressores da germinação obtidos com extratos de carqueja (Silva \& Carvalho, 2009). Os taninos hidrolisáveis são conhecidos alelopaticamente por atuarem como inibidores da germinação de sementes, do crescimento de plantas e das bactérias fixadoras de nitrogênio e das nitrificantes (Castro \& Ferreira, 2001).

O óleo de Melaleuca alternifolia possui composição química complexa, que agindo em sinergismo, confere a este óleo um amplo espectro de ação (Garcia et al. 2009). Estudos farmacológicos confirmaram propriedades antisséptica, bactericida, cicatrizante, expectorante, fungicida e antiviral no óleo desta espécie, sendo popularmente utilizado em tratamentos de cortes, ferimentos e infecções cutâneas bacterianas e fúngicas (Corazza, 2004). Quanto aos efeitos alelopáticos em nível de germinação de sementes, a aplicação de óleo de melaleuca no tratamento prévio de sementes de girassol proporcionou, além de controle mais eficiente do fungo Alternaria spp. na sanidade de sementes, maior emergência de plântulas, sob condições controladas de laboratório (Mariano et al., 2014). No presente experimento, a germinação de capim annoni foi inibida pela adição deste óleo.

Corroborando com os resultados deste trabalho, efeitos inibitórios da germinação já foram atribuídos ao óleo essencial de melaleuca por outros pesquisadores. Seu óleo puro inibiu a germinação das sementes de milho e preservou as sementes contra-ataques de microrganismos (Lima \& Freitas, 2016). Além disso, o extrato de melaleuca interferiu na germinação de Urochloa brizantha (Hochst. ex A. Rich.) R.D. Webster, podendo ser útil para o manejo dessa espécie vegetal (Queiroz et al., 2016). Alguns terpenos, encontrado em grandes quantidades em espécies de Myrtaceae, são apontados como possíveis responsáveis pelo efeito fitotóxico do óleo de melaleuca, especialmente terpinenol-4 que, segundo revisão de Monteiro et al. (2013), é o quimiotipo mais abundante na melaleuca, seguido de gamaterpineno, alfa-terpineno e 1,8-cineol presente nas folhas, ápice e ramos de plantas cultivadas no Brasil.

O louro (Laurus nobilis) é uma espécie aromática bastante conhecida de Lauraceae, utilizada pelas indústrias alimentícia, farmacêutica e cosmética. Dentre essas aplicações, as propriedades antimicrobianas do óleo essencial de louro são já bastante exploradas (Fiorini et al., 1997; Ozcan \& Erkmen, 2001; Sangun et al., 2007; Ozcan et al., 2010).

Seguindo outra linha de estudo, efeito deste óleo na germinação de sementes foi demonstrado por Rossi et al. (2012) que, em concentrações elevadas (50\%) reduziu a germinação de trigo e milho em 92,8 e 85,7\%, respectivamente. Embora neste trabalho 
tenham sido utilizadas concentrações de óleo inferiores às empregadas por Rossi et al. (2012), foi possível observar redução significativa na germinação de capim annoni. Análises fitoquímicas têm revelado a presença de terpenoides, antocianinas e glicosídeos no óleo de louro (Patrakar et al., 2012), podendo sugerir que os terpenoides sejam os atores das relações alelopáticas desta espécie.

Os resultados deste trabalho, embora ainda iniciais, abrem novas perspectivas no tão desafiador controle do capim annoni, uma vez que a identificação de espécies com potencial alelopático de controle possibilita o desenvolvimento de estratégias de manejo que poderiam incluir tanto o uso como cobertura vegetal ou mesmo na formulação de bioprodutos. Além disso, estratégias como estas contribuem para a redução do uso de insumos químicos com potencial poluente, o que hoje é um apelo tanto do ponto de vista ambiental quanto econômico.

\section{Agradecimentos}

\section{Apoio Financeiro: CNPq e FAPERGS}

\section{Referências}

1. ALFAYA, H., SUÑÉ, L. N. P. \& SIQUEIRA, C. M. G. 2002. Efeito da amonização com ureia sobre os parâmetros de qualidade do feno do capim-annoni 2 (Eragrostis plana Nees). Revista Brasileira de Zootecnia, 31(2): 842-851.

2. BALBI-PEÑA, M. I., BECKER, A., STANGARLIN, J. R., FRANZENER, G., LOPES, M. C. \& SCHWAN-ESTRADA, K. R. F. 2006. Controle de Alternaria solani em tomateiro por extratos de Cúrcuma longa e curcumina - II Avaliação in vitro. Fitopatologia Brasileira, 1: 10-14.

3. BONA, C. M.; BIASI, L. A., NAKASHIMA, T., ZANETTE, F. \& CORRÊA-JÚNIOR, C. 2002. Carqueja: Cultive esta ideia. Curitiba: SEAB-PR, 18p.

4. CASTRO, H. G. \& FERREIRA, F. A. Contribuição ao estudo das plantas medicinais (Baccharis genistelloides). Viçosa: Ed. UFV, 2001.

5. BRASIL. 2009. Ministério da Agricultura, Pecuária e Abastecimento. Regras para análise de sementes. Ministério da Agricultura, Pecuária e Abastecimento. Secretaria de Defesa Agropecuária. Brasília, DF: Mapa/ACS, 395p.

6. CICCONET, N., ALBA, J. M. F., SPIRONELLO, R. L. \& CUNHA, H. 2015. Mapeamento de Eragrostis plana Nees (capim-annoni) por meio de imagens orbitais. Revista do Departamento de Geografia - USP, 29: 20-33.

7. CORAZZA, S. 2004. Aromocologia. Uma ciência de muitos cheiros. $3^{\circ}$ Ed. São Paulo, SP. Senac, $540 \mathrm{p}$.

8. DEPINÉ, C. Efeito alelopático de carqueja sobre a germinação de plantas daninhas. 2003. 43 f. Monografia (Bacharelado em Ciências Biológicas) - Núcleo de Ciências Biológicas e da Saúde. Centro Universitário Positivo, Curitiba, 2003.

9. Ens, E. J., Bremner, J. B., French, K. \& Korth, J. 2009. Identification of volatile compounds released by roots of an invasive plant, bitou bush (Chrysanthemoides monilifera spp. rotundata), and their inhibition of native seedling growth. Biological Invasions, 11: 275287. 
10. FAROOQ, M., JABRAN, K., ZAHID, A. C., WAHID, A. \& SIDDIQUE, K. H. M. 2011. The role of allelopathy in agricultural pest management. Pest Management Science, 67(5): 493-506.

11. FERREIRA, A. G. \& BORGHETTI, F. 2004. Germinação: do básico ao aplicado. Porto Alegre: Artmed, $323 \mathrm{p}$.

12. FERREIRA, N. R., MEDEIROS, R.B. \& SOARES, G. L.G. 2008. Potencial alelopático do capimannoni-2 (Eragrostis plana Nees) na germinação de sementes de gramíneas perenes estivais. Revista Brasileira de Sementes, 30(2): 043-050.

13. FERREIRA, A.T. Uma "exótica" no campo: o capim annoni-2 e suas influências na produção pecuária da coxilha de São Rafael, no município de Quaraí - RS. 56 f. Trabalho de conclusão apresentado como requisito parcial para a obtenção do título de Tecnólogo em Planejamento e Gestão para o desenvolvimento Rural, UFRGS. 2011a.

14. FERREIRA, D. F. 2011b. Sisvar: a computer statistical analysis system. Ciência e Agrotecnologia, 35(6): 1039-1042.

15. FIORINI, C., FOURASTE, I. DAVID, B. \& BESSIERE, M. 1997. Composition of the flower, leaf and stem essential oils from Laurus nobilis. L. Flavour and Fragrance Journal, 12(2): 91-93.

16. FOCHT, T. Ecologia e dinâmica do capim-annoni-2 (Eragrostis plana Nees), uma invasora dos campos sulinos: prevenção da sua expansão. 154 f. Tese de Conclusão de Curso Universidade Federal do Rio Grande do Sul - Programa de Pós-Graduação em Ecologia. Porto Alegre, 2008.

17. GARCIA, C. C., GERMANO, C., OSTI, N. M. \& CHORILLI, M. 2009. Desenvolvimento e avaliação da estabilidade físico-química de formulações de sabonete líquido íntimo acrescidas de óleo de Melaleuca. Revista Brasileira de Farmacologia, 90(3): 236-240.

18. GOULART, I. C. G. R., MEROTTO JUNIOR, A., PEREZ, N.B. \& KALSING, A. 2009. Controle de capim-annoni-2 (Eragrostis plana) com herbicidas pré-emergentes em associação com diferentes métodos de manejo do campo nativo. Planta Daninha, 27(1): 181-190.

19. KIRKMAN, K.P. \& MORRIS, C.D. 2003. Ecology and dynamics of Eragrostis curvula and Eragrostis plana with a view of controlling their spread in natural grasslands. In: Proceedings of the VII International Rangelands Congress. 26 July-1 August 2003, Durban, South Africa. Proceedings... Durban, South Africa: International Grassland Society, 2003. 138p.

20. LIMA, A. A. \& FREITAS, W. L. C. Efeito alelopático do óleo essencial de Melaleuca alternifolia Cheel sobre a germinação de sementes de milho (Zea mayz L.). VII Congresso de Iniciação Científica da FEPI - Pesquisa Científica, Oportunidades e Desafios. 2016.

21. MAGUIRE, J. D. 1962. Speed of germination-aid in selection and evaluation for seedling emergence and vigor. Crop Science, 2(1): 176-177.

22. MARIANO, D. C.; GIEBELMEIER, C. G.; AlBUQUERQUE, G. D. P.; SILVA, C. R.; OKUMURA, R. S. 2014. Uso do óleo de Melaleuca alternifolia no tratamento de sementes de girassol. Enciclopédia Biosfera - Centro Científico Conhecer, 10(18): 2961-2975. 
23. MEDEIROS, R.B. \& FOCHT, T. 2007. Invasão, prevenção, controle e utilização do capimannoni (Eragrostis plana Nees) no Rio Grande do Sul. Pesquisa Agropecuária Gaúcha, 13(1-2): 105-114.

24. MONTEIRO, M.H.D.A., MACEDO, H.W. de; JUNIOR, A. da S. \& PAUMGARTTEN, F.J.R. 2013. Óleos essenciais terapêuticos obtidos de espécies de Melaleuca L. (Myrtaceae Juss.). Farmanguinhos/Fiocruz. Revista Fitos Eletrônica, [S.I.], 8(1): 19-32.

25. NACHTIGAL, G. F., NEUMANN, E. L. F., COSTA, F. A. \& GARLICH, N. 2009. Ocorrência de ferrugem associada ao capim-annoni-2 (Eragrostis plana Nees) na região de fronteira da metade sul do RS. Revista Brasileira de Agroecologia, 4(2): 12-31.

26. OZCAN, M. \& ERKMEN, O. 2001. Antimicrobial activity of the essential oils of Turkish plant spices. European Food Research and Technology, 212: 658-660.

27. OZCAN, B., ESEN, M., SANGUN, M. K., COLERI, A. \& CALISKAN, M. 2010. Effective antibacterial and antioxidant properties of methanolic extract of Laurus nobilis seed oil. Journal of Environmental Biology, 31(5): 637-641.

28. PATRAKAR, R., MANSURIYA, M. \& PATIL, O. 2012. Phytochemical and Pharmacological Review on Laurus nobilis. International Journal of Pharmaceutical and Chemical Sciences, 1(2): 595-602.

29. PINHO, D. S. de, STURBELLE, R. T., MARTINO-ROTH, M. G. \& GARCIAS, G. L. 2010. Avaliação da atividade mutagênica da infusão de Baccharis trimera (Less.) DC. em teste de Allium cepa e teste de aberrações cromossômicas em linfócitos humanos. Revista Brasileira de Farmacognosia, 20: 165-170.

30. QUEIROZ, R. L., PIRES, V., BARTELEGA, A., CARVALHO, J. W. M. \& SERAFIM A. J. 2016. Avaliação de extrato de Melaleuca alternifolia (Cheel) na germinação de Brachiaria brizantha. Revista Fitos, 10(4): 375-547.

31. ROSSI, E., COSIMI, S. \& LONI, A. 2012. Bioactivity of essential oils from Mediterranean plants: Insecticidal properties on Sitophilus zeamais and effects on seed germination. Journal of Entomology, 9(6): 403-412.

32. SANGUN, M. K., AYDIN, E. TIMUR, M., KARADENIZ, H., CALISKAN, M. \& OZKAN, A. 2007. Comparison of chemical composition of the essential oil of Laurus nobilis L. leaves and fruits from different regions of Hatay, Turkey. Journal of Environmental Biology, 28: 731733.

33. SILVA, A. G. \& CARVALHO, R. I. N. 2009. Efeito alelopático de extratos de carqueja (Baccharis trimera) e confrei (Symphytum officinale) em sementes e plântulas de girassol. Revista Acadêmica: Ciências Agrárias e Ambientais, 7(1): 23-32.

34. SOUZA FILHO, A. P. S., GUILHON, G. M. S. P., ZOGHBI, M. G. B. \& CUNHA, R. L. 2009. Análise comparativa do potencial alelopático do extrato hidroalcoólico e do óleo essencial de folhas de Cipó-d'alho (Bignoniaceae). Planta Daninha, 27(4): 647-653.

35. TAKATSUKA, F. S., SILVA, I. D., OLIVEIRA, M. F., CZEPAK, C., OLIVEIRA, C. M. A. \& CUNHA, M. G. 2003. Efeito do óleo essencial de açafrão (Curcuma longa) sobre o 
desenvolvimento micelial de fungos. In: $36^{\circ}$ CONGRESSO BRASILEIRO DE FITOPATOLOGIA, Anais... Uberlândia, Brasil, v.28, p.361.

36. TAYLOR, L. P. \& GROTEWOLD, E. 2005. Flavonoids as developmental regulators. Current Opinion in Plant Biology, 8(3):317-323.

37. VITTI, A. M. S. \& BRITO, J. O. Óleo essencial de eucalipto. Piracicaba: IPEF, 2003. 26 p. (Documentos florestais).

38. WEIR, T. L., PARK, S. W. \& VIVANCO, J. M. 2004. Biochemical and physiological mechanisms mediated by allelochemicals. Current Opinion in Plant Biology, 7(4): 472479.

39. ZENNI, R.D. \& ZILLER, S.R. 2011. An overview of invasive plants in Brazil. Revista Brasileira de Botânica, 34: 431-446.

\section{Anexos}

Tabela 1: Primeira contagem de germinação (\%), in vitro, de Eragrostis plana em exposição a diferentes concentrações de óleos essenciais de espécies vegetais, após sete dias de instalação.

\begin{tabular}{|c|c|c|c|c|c|c|c|c|}
\hline & Controle & C. álcool & 0,001 & 0,005 & 0,01 & 0,05 & 0,1 & CV \\
\hline Alecrim & $57,47 a b$ & $53,17 \mathrm{ab}$ & 59,99 a & $56,85 a b$ & $51,37 \mathrm{ab}$ & $47,89 \mathrm{~b}$ & $47,89 \mathrm{~b}$ & 9,71 \\
\hline Canela & $60,77 \mathrm{a}$ & $58,27 \mathrm{ab}$ & $64,68 \mathrm{a}$ & $53,35 a b$ & $42,67 \mathrm{~b}$ & $49,88 \mathrm{ab}$ & $55,02 a b$ & 12,59 \\
\hline Capim limão & $54,98 \mathrm{a}$ & $55,00 \mathrm{a}$ & 56,99 a & $55,62 \mathrm{a}$ & $60,38 \mathrm{a}$ & 57,16 a & 49,67 a & 11,21 \\
\hline Carqueja & $58,89 a$ & $62,80 \mathrm{a}$ & $55,30 a b$ & $58,73 \mathrm{a}$ & $57,12 \mathrm{a}$ & $49,78 a b$ & $41,50 \mathrm{~b}$ & 11,59 \\
\hline Cipreste & $69,04 \mathrm{a}$ & $69,04 \mathrm{a}$ & $68,08 \mathrm{a}$ & $68,22 \mathrm{a}$ & $70,26 \mathrm{a}$ & $65,06 \mathrm{a}$ & $68,09 \mathrm{a}$ & 5,63 \\
\hline Citronela & $56,85 a b$ & $53,90 a b c$ & $55,25 a b$ & $60,70 \mathrm{a}$ & $54,48 a b c$ & $40,93 \mathrm{c}$ & $43,81 \mathrm{bc}$ & 11,40 \\
\hline $\begin{array}{l}\text { Eucalipto } \\
\text { citriodora }\end{array}$ & 58,08 a & $57,11 a$ & $61,01 \mathrm{a}$ & $55,62 a$ & 59,63 a & $55,72 a$ & $54,42 \mathrm{a}$ & 9,50 \\
\hline $\begin{array}{c}\text { Eucalipto } \\
\text { grandis }\end{array}$ & $54,98 \mathrm{a}$ & $57,11 a$ & $58,29 a$ & $55,62 a$ & $57,66 \mathrm{a}$ & $55,65 a$ & $55,11 a$ & 9,49 \\
\hline Louro & $61,36 \mathrm{ab}$ & $57,60 a b$ & $58,08 a b$ & $50,27 \mathrm{bc}$ & $62,97 a$ & $50,90 \mathrm{bc}$ & $44,99 \mathrm{c}$ & 9,19 \\
\hline Melaleuca & $61,00 \mathrm{a}$ & $60,08 \mathrm{a}$ & 57,09 a & 58,08 a & $39,70 \mathrm{~b}$ & $35,03 \mathrm{~b}$ & $33,65 \mathrm{~b}$ & 10,65 \\
\hline Pinus & $56,85 a$ & $57,68 a$ & $56,32 \mathrm{a}$ & $56,35 a$ & $60,77 a$ & $50,28 a$ & $53,17 a$ & 8,79 \\
\hline
\end{tabular}


Tabela 2: Germinação acumulada (\%), in vitro, de Eragrostis plana em exposição a diferentes concentrações de óleos essenciais de espécies vegetais, após 14 dias de instalação.

\begin{tabular}{c|c|c|c|c|c|c|c|c} 
& Controle & C. álcool & 0,001 & 0,005 & 0,01 & 0,05 & 0,1 & $\mathrm{CV}$ \\
\hline Alecrim & $68,29 \mathrm{a}$ & $62,14 \mathrm{ab}$ & $65,76 \mathrm{a}$ & $58,08 \mathrm{bc}$ & $53,18 \mathrm{~cd}$ & $49,33 \mathrm{~d}$ & $54,37 \mathrm{~cd}$ & 5,65 \\
\hline Canela & $81,22 \mathrm{a}$ & $76,71 \mathrm{ab}$ & $74,93 \mathrm{ab}$ & $70,82 \mathrm{ab}$ & $65,36 \mathrm{ab}$ & $63,89 \mathrm{ab}$ & $60,74 \mathrm{~b}$ & 11,12 \\
\hline Capim limão & $66,20 \mathrm{a}$ & $63,62 \mathrm{a}$ & $62,26 \mathrm{a}$ & $56,85 \mathrm{a}$ & $60,38 \mathrm{a}$ & $58,34 \mathrm{a}$ & $53,95 \mathrm{a}$ & 9,68 \\
\hline Carqueja & $82,05 \mathrm{a}$ & $71,65 \mathrm{ab}$ & $65,16 \mathrm{bc}$ & $60,70 \mathrm{bc}$ & $58,91 \mathrm{bc}$ & $51,53 \mathrm{~cd}$ & $43,28 \mathrm{~d}$ & 9,66 \\
\hline Cipreste & $74,11 \mathrm{a}$ & $80,82 \mathrm{a}$ & $80,12 \mathrm{a}$ & $84,12 \mathrm{a}$ & $81,35 \mathrm{a}$ & $74,18 \mathrm{a}$ & $72,05 \mathrm{a}$ & 10,03 \\
\hline Citronela & $58,29 \mathrm{a}$ & $55,76 \mathrm{a}$ & $56,42 \mathrm{a}$ & $63,54 \mathrm{a}$ & $57,33 \mathrm{a}$ & $49,63 \mathrm{a}$ & $49,67 \mathrm{a}$ & 10,85 \\
\hline $\begin{array}{c}\text { Eucalipto } \\
\text { citriodora }\end{array}$ & $67,46 \mathrm{a}$ & $62,97 \mathrm{ab}$ & $63,54 \mathrm{ab}$ & $57,45 \mathrm{~b}$ & $62,24 \mathrm{ab}$ & $62,89 \mathrm{ab}$ & $64,24 \mathrm{ab}$ & 6,28 \\
\hline Eucalipto grandis & $68,29 \mathrm{a}$ & $68,21 \mathrm{a}$ & $61,45 \mathrm{a}$ & $60,70 \mathrm{a}$ & $62,14 \mathrm{a}$ & $62,14 \mathrm{a}$ & $60,70 \mathrm{a}$ & 5,33 \\
\hline Louro & $69,51 \mathrm{a}$ & $68,07 \mathrm{a}$ & $58,77 \mathrm{abc}$ & $50,27 \mathrm{~cd}$ & $65,06 \mathrm{ab}$ & $53,37 \mathrm{bcd}$ & $42,69 \mathrm{~d}$ & 10,69 \\
\hline
\end{tabular}

Tabela 3: Índice de velocidade de germinação, in vitro, de Eragrostis plana em exposição a diferentes concentrações de óleos essenciais de espécies vegetais.

\begin{tabular}{c|c|c|c|c|c|c|c|c} 
& Controle & $\mathrm{C}$. álcool & 0,001 & 0,005 & 0,01 & 0,05 & 0,1 & $\mathrm{CV}$ \\
\hline Alecrim & $5,94 \mathrm{a}$ & $4,83 \mathrm{abc}$ & $5,58 \mathrm{ab}$ & $5,48 \mathrm{abc}$ & $4,71 \mathrm{abc}$ & $4,28 \mathrm{bc}$ & $3,92 \mathrm{c}$ & 14,51 \\
\hline Canela & $5,48 \mathrm{a}$ & $4,48 \mathrm{abc}$ & $5,08 \mathrm{ab}$ & $3,81 \mathrm{bc}$ & $2,95 \mathrm{c}$ & $3,74 \mathrm{bc}$ & $3,98 \mathrm{abc}$ & 16,57 \\
\hline Capim limão & $5,17 \mathrm{a}$ & $5,40 \mathrm{a}$ & $5,56 \mathrm{a}$ & $4,94 \mathrm{a}$ & $5,06 \mathrm{a}$ & $4,48 \mathrm{a}$ & $4,05 \mathrm{a}$ & 19,34 \\
\hline Carqueja & $5,44 \mathrm{a}$ & $4,89 \mathrm{ab}$ & $4,20 \mathrm{abc}$ & $4,66 \mathrm{ab}$ & $3,77 \mathrm{abc}$ & $3,27 \mathrm{bc}$ & $2,70 \mathrm{c}$ & 20,24 \\
\hline Cipreste & $6,08 \mathrm{a}$ & $5,95 \mathrm{a}$ & $6,10 \mathrm{a}$ & $6,58 \mathrm{a}$ & $6,47 \mathrm{a}$ & $5,91 \mathrm{a}$ & $5,90 \mathrm{a}$ & 5,33 \\
\hline Citronela & $5,55 \mathrm{a}$ & $4,97 \mathrm{a}$ & $5,13 \mathrm{a}$ & $5,97 \mathrm{a}$ & $5,00 \mathrm{ab}$ & $3,83 \mathrm{~b}$ & $3,74 \mathrm{~b}$ & 14,18 \\
\hline $\begin{array}{c}\text { Eucalipto } \\
\text { citriodora }\end{array}$ & $5,07 \mathrm{ab}$ & $5,03 \mathrm{ab}$ & $5,21 \mathrm{a}$ & $5,17 \mathrm{ab}$ & $5,44 \mathrm{a}$ & $4,62 \mathrm{ab}$ & $4,26 \mathrm{a}$ & 8,09 \\
\hline $\begin{array}{c}\text { Eucalipto } \\
\text { grandis }\end{array}$ & $5,23 \mathrm{ab}$ & $5,69 \mathrm{a}$ & $5,11 \mathrm{ab}$ & $4,96 \mathrm{ab}$ & $4,81 \mathrm{ab}$ & $4,34 \mathrm{~b}$ & $4,33 \mathrm{~b}$ & 10,80 \\
\hline Louro & $5,98 \mathrm{a}$ & $5,14 \mathrm{ab}$ & $5,30 \mathrm{ab}$ & $4,86 \mathrm{ab}$ & $4,97 \mathrm{ab}$ & $4,87 \mathrm{ab}$ & $4,0 \mathrm{~b}$ & 11,84 \\
\hline Melaleuca & $5,54 \mathrm{a}$ & $5,60 \mathrm{a}$ & $3,73 \mathrm{bc}$ & $4,57 \mathrm{ab}$ & $3,00 \mathrm{bc}$ & $2,73 \mathrm{c}$ & $2,39 \mathrm{c}$ & 18,88 \\
\hline Pinus & $5,42 \mathrm{a}$ & $5,33 \mathrm{a}$ & $5,24 \mathrm{a}$ & $5,03 \mathrm{a}$ & $5,22 \mathrm{a}$ & $5,10 \mathrm{a}$ & $4,69 \mathrm{a}$ & 10,27
\end{tabular}

*Médias seguidas pela mesma letra, na linha, não diferem entre si pelo teste de Tukey a $5 \%$ de probabilidade. $\mathrm{CV}=$ Coeficiente de variação. 